\title{
Efficient design of meganucleases using a machine learning approach
}

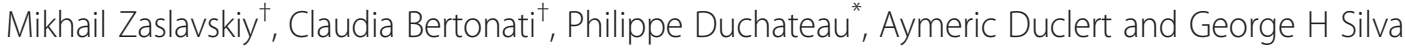

\begin{abstract}
Background: Meganucleases are important tools for genome engineering, providing an efficient way to generate DNA double-strand breaks at specific loci of interest. Numerous experimental efforts, ranging from in vivo selection to in silico modeling, have been made to re-engineer meganucleases to target relevant DNA sequences.

Results: Here we present a novel in silico method for designing custom meganucleases that is based on the use of a machine learning approach. We compared it with existing in silico physical models and high-throughput experimental screening. The machine learning model was used to successfully predict active meganucleases for 53 new DNA targets.

Conclusions: This new method shows competitive performance compared with state-of-the-art in silico physical models, with up to a fourfold increase in terms of the design success rate. Compared to experimental high-throughput screening methods, it reduces the number of screening experiments needed by a factor of more than 100 without affecting final performance.
\end{abstract}

\section{Background}

Genome engineering (GE) focuses on the modification of genomes in living organisms at specific loci of interest. Examples of such modifications include insertion of a new gene into the genome, inactivation of existing genes via disruption of their sequences and replacement of a malfunctioning gene with a corrected version. GE has demonstrated its utility in basic research as well as in many industrial applications, for instance in agriculture and therapeutics [1-3]. One approach to GE is based on the use of sequence-specific nucleases to trigger DNA modifications by generating DNA double-strand breaks at the locus of interest. Currently, the four most frequently used tools in GE to generate targeted DNA cleavage are transcription activator-like effector nucleases $\left(T_{A L E N}{ }^{a}\right)$ [4], CRISPR nuclease complexes [5,6], zinc-finger nucleases (ZFN) $[4,7,8]$ and meganucleases $(\mathrm{MN})[9,10]$. These proteins have different properties (size, origin etc.), making it possible to match them with specific applications $[3,11]$.

Meganucleases are naturally occurring endonucleases characterized by large recognition sites (12 bp or more), which are almost unique in most genomes. The large

\footnotetext{
* Correspondence: philippe.duchateau@cellectis.com

${ }^{\dagger}$ Equal contributors

Research and Development department, Cellectis, 8 rue de la Croix Jarry,

Paris 75013, France
}

recognition sites makes MNs perfect tools for GE, but unfortunately the number of naturally occurring MNs is quite limited and is not nearly sufficient to cover all potentially interesting loci. Therefore there was a strong need for a method that would allow us to redesign existing MNs to cut new DNA sequences. Existing redesign techniques include the creation of fusion chimeras from existing MN domains [12-14] and alteration of $\mathrm{MN}$ specificity via direct mutation of protein residues in the DNA binding scaffold [15-25]. One of the most used starting scaffolds for the design of new artificial MNs is I-CreI, a member of the LAGLIDADG family, the largest of five known families of MNs [26]. I-CreI is a homodimeric endonuclease cutting a $22 \mathrm{bp}$ pseudopalindromic target (Figure 1A) with at least 25 known structures in the RCSB PDB [27] showing an alpha-beta(2)-alpha-beta (2)-alpha fold [28] (see Figure 1B). Figure 1C illustrates which residues participate in the binding of DNA [29]. In each monomer, residues R70 make direct contacts with base \pm 3 and \pm 4 , Q44 with base \pm 4 , and R68 with base \pm 5 , while Q26 and K28 make direct contacts with bases \pm 6 and \pm 7 s respectively. Positions \pm 10 make direct contacts with residue Y33 and \pm 9 with Q38 and N30. The base pairs at positions \pm 1 and \pm 2 (2N4 region) do not make direct contacts with any of the protein residues. Based on this interaction map, we previously 


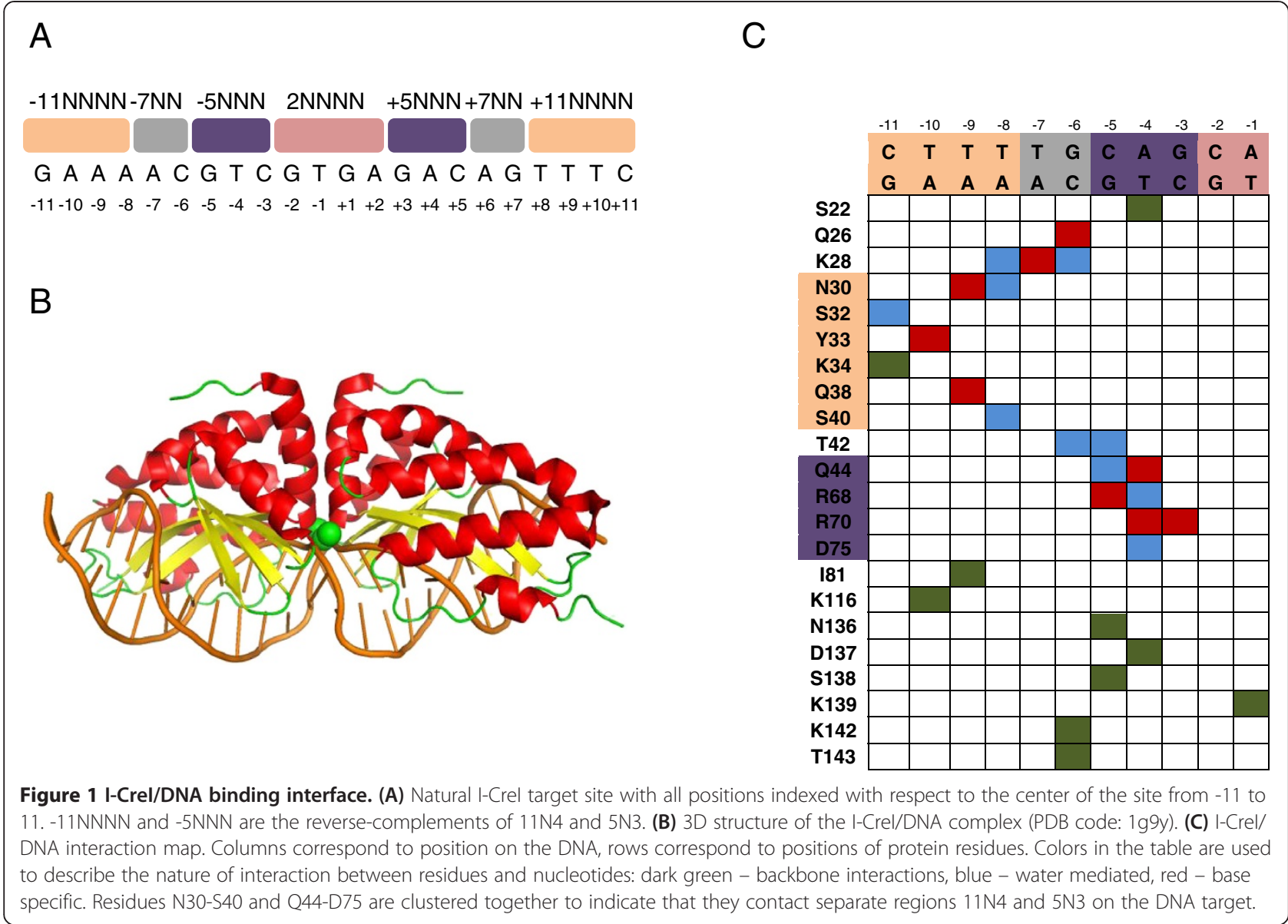

defined four regions on the I-CreI target: the central 2N4 part of the recognition site (positions -2:2), where I-CreI cuts the DNA and three regions $\pm 11 \mathrm{~N} 4, \pm 7 \mathrm{~N} 2$, $\pm 5 \mathrm{~N} 3$ which constitute the binding sites [15-17].

Much effort has been made in the past to engineer I-CreI [15-21]. We formerly reported a successful combinatorial approach relying on the division of the I-CreI/DNA interface into separate clusters of amino acids with different DNA recognition regions. Namely, the fact that there is no intersection between groups of residues binding the $5 \mathrm{~N} 3$ and $11 \mathrm{~N} 4$ regions (see Figure $1 \mathrm{C}$ ) makes it possible to screen for proteins binding target variants in the $5 \mathrm{~N} 3$ and $11 \mathrm{~N} 4$ regions independently and then combine the mutations from these two protein sets to cut a hybrid 11N4-5N3 target [15-17]. While conceptually the combination of different clusters allows efficient MN design, one still needs to screen hundreds of molecules to find the optimal MN. In [16], an assay based on single-strand annealing (SSA) was developed to perform such high-throughput screening as a routine assay. Another example of a semi-rational experimental approach is based on the sequential design of I-OnuI derived MNs [22]. Here, we present a new method for $\mathrm{MN}$ engineering that significantly reduces the number of molecules screened without reducing the final success rate.

Several studies have described the possibility of engineering MNs using atomistic molecular modeling software such as FoldX [19] and Rosetta [18,20,21]. These software packages build optimal MN structures by minimizing the binding energy of protein-DNA complexes. Most reported studies have limited the use of these packages mainly to the prediction of MNs for single base substitutions, but in [18] the authors reported successful MN prediction on targets with up to three base substitutions.

Diverse applications of machine learning (ML) approaches [30-36] have positioned machine learning techniques as promising tools for solving complex biological problems. Some of these applications, such as how to predict transcription factors, bear many similarities with the design of MNs. A necessary condition for the application of a ML approach is the existence of data from which a ML algorithm can learn a model. Over the years, we have gathered data on the cleavage activity of several hundreds of thousands of MN-DNA target pairs, which we used to train a machine learning model. 
In this manuscript we present a new efficient method of $\mathrm{MN}$ design based on machine learning techniques. This strategy presents the advantage of a competitive success rate compared to that of experimental combinatorial high-throughput screening, while significantly reducing the number of screened molecules by several orders of magnitude and at the same time significantly outperforming alternative in silico models such as Rosetta and FoldX. The experimental validation of the ML approach lead to the successful design of MNs for 53 new DNA targets.

\section{Results}

\section{Cross-validation experiments}

The principal dataset used to train/cross-validate the machine learning model and to compare the performance of various in silico models consisted of 251 pseudopalindromic (reduction of non-palindromic targets to pseudopalindromic is described in [17]) 22 bp DNA targets screened according to a combinatorial process (see "Materials \& methods") giving in total 293k protein-DNA pairs of known activity (each target had at least one active protein).

In the first series of experiments we studied how in silico methods (ML approach and physical models) performed on the combinatorial dataset by doing cross-validation experiments (a detailed description of cross-validation scheme is given in Additional file 1: Figure S1). To assess the quality of model predictions, we computed several performance scores for each target: AUC score, Top10 score, and \% Top10. Finally, the average value of each score over all targets from the test set was used as a global performance measure of in silico models (hereinafter we will use the simplified notations AUC, Top10, and \%Top10 to denote the overall average values of each score).

Figure 2 presents the cross-validation performance scores (Top10\% and AUC) for the following models: FoldX (Fx),
Rosetta (Rt), Mact (machine learning model trained only on the cleavage activities of the p5N3 and p11N4 building modules), SeqMact (ML model trained on the cleavage activities of building modules plus target and protein sequences), SeqMactFxStr (ML model trained on all available features such as cleavage activities of building modules, target and protein sequences, FoldX scores and protein-DNA interaction maps). Values of Top10 score are given in Additional file 1: Figure S6. FoldX and Rosetta did not use the information available in the training set; they made their predictions by estimating the binding energy of the proteinDNA couple from a physical model. For a more detailed description of ML models, FoldX and Rosetta, see the corresponding sections of "Materials and methods".

Overall Mact had an AUC score of around 0.66 and was able to predict at least one positive protein in the top 10 for about $20 \%$ of targets. Remarkably, the performance of the physical models (Fx and Rt) matched that of Mact. It is worth noting that Fx/Rt do not use information on module cleavage activities and can be used $a b$ initio without any preliminary steps (i.e. screening of module libraries). However, when we added the information on protein and target sequences into the ML model (SeqMact), we obtained a significantly better success rate, predicting at least one positive mutant in the top 10 for about $80 \%$ of targets, with an AUC score of 0.9 (Additional file 1: Figure S13 shows the average ROC curve computed over test targets). The substantial difference in the success rates of Mact and SeqMact suggested that combining the best modules was not sufficient to get an active combined mutant; we also had to take into account protein and target sequence composition. Another important conclusion was that by learning sequence patterns specific to active and non-active proteins, we could obtain much better predictions than by exploiting a general physical model. Interestingly, when we combined SeqMact and Fx into a more general ML model (SeqMactFxStr)
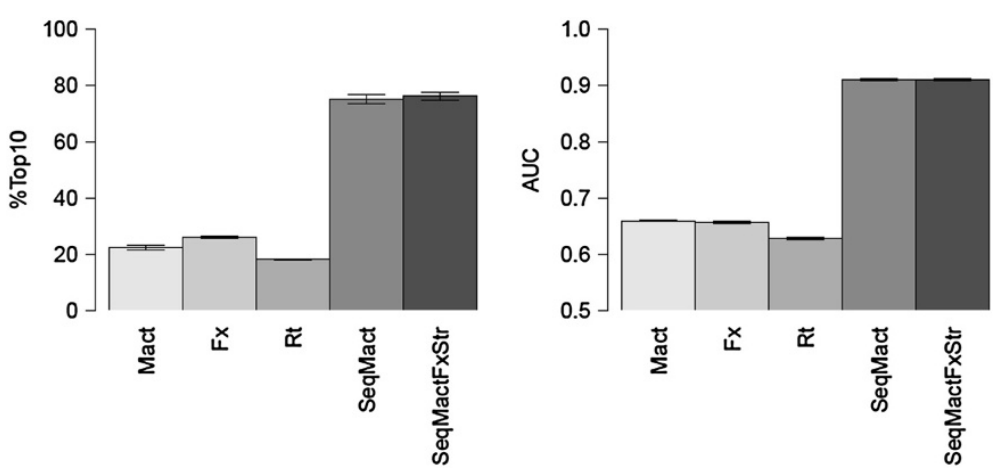

Figure 2 Cross-validation performance of various in silico methods. (Left) \%Top10 — percentage of targets with at least one positive molecule in Top10 ranked, (Right) AUC - AUC score (see Material and Methods) Mact - predictions made on the basis of module cleavage activities, Fx — FoldX score, Rt — Rosetta score, SeqMact — protein/target sequences + module cleavage activities, SegMactFxStr — all features combined (sequences + module cleavage activities + FoldX scores and interactions). Error bars are estimated from 30 independent cross-validation experiments. 
trained on sequence and structural features, we did not observe any improvement even though the two methods' final protein rankings were not always the same.

Machine learning performance scores presented in Figure 2 correspond to the case when all available data in the training set were used to train the model (on average screening results on $226=0.9 * 251$ targets). Figure 3 (Left) shows how Top10\% (AUC and TopN are presented in Additional file 1: Figure S7) of Mact and SeqMact varies with the size of the training set, with only $10-20$ targets $(\sim 10 \%$ of the actual traing set) SeqMact already matches the performance of FoldX and with 60 targets, it almost triples the performance. To make sure that SeqMact generalizes well on targets which are significantly different from what we already have in the training set, we repeated the previous experiment but this time instead of random subsampling of targets from the training set, we kept only those that had at least 2 and 3 base pair difference with all test targets (1 bp difference is the default situation where we use the entire training split). Figure 3 (Right) shows how the performance varied with the minimal allowed distance between training and test sets, we observe a drop in performance when the minimal distance (red circles) is increased. However the decrease is almost identical when we randomly sample an equivalent number of targets meaning that the model predicts well on significantly different targets and the observed drop in performance is due to the reduced size of the training set. Additional file 1: Figure S8 presents the distribution of all possible MN targets with respect to their distance from our training set. A majority of targets were at most 3 nucleotides distant from the training set, indicating that we could safely apply our method to almost all potential MN targets.

Another important question is how many molecules we needed to screen in order to have at least one positive mutant. Additional file 1: Figure S9 shows how the success rate of in silico approaches varied with the size of the screening pool. Ten molecules seemed to provide a good compromise between the number of molecules tested and the corresponding success rate; six molecules were enough to have on average one active molecule and at least one active molecule for more than $50 \%$ of the targets tested.

\section{Key features in machine learning model}

In this section we address why SeqMact was more efficient than Mact. As described in "Cross-validation performance scores", SeqMact can be seen as an extended version of Mact with additional features describing protein and target sequences. To determine whether any particular group of features contributed most to the performance boost, we tested alternative versions of SeqMact trained on several subgroups of features:

- SM-5 (SM-11 respectively): features encoding the p5N3 (p11N4 respectively) part of proteins and DNA sequences; no interactions between features; the relative performance of this model with respect to Mact reflected the importance of the p5N3 (p11N4 respectively) part of the sequences;

- SM-5_11: union of features from the two previous models; no interactions between features; the relative performance of this model with respect to SM-5 and SM-11 showed whether the combination of both parts (p5N3 and p11N4) improved performance with respect to the individual use of each part;

- SM-M2M: all features from the SM-5_11 model plus interactions between features encoding protein sequences; the relative performance of this model with respect to SM-5_11 reflected the impact of simultaneous protein mutations, i.e. whether there
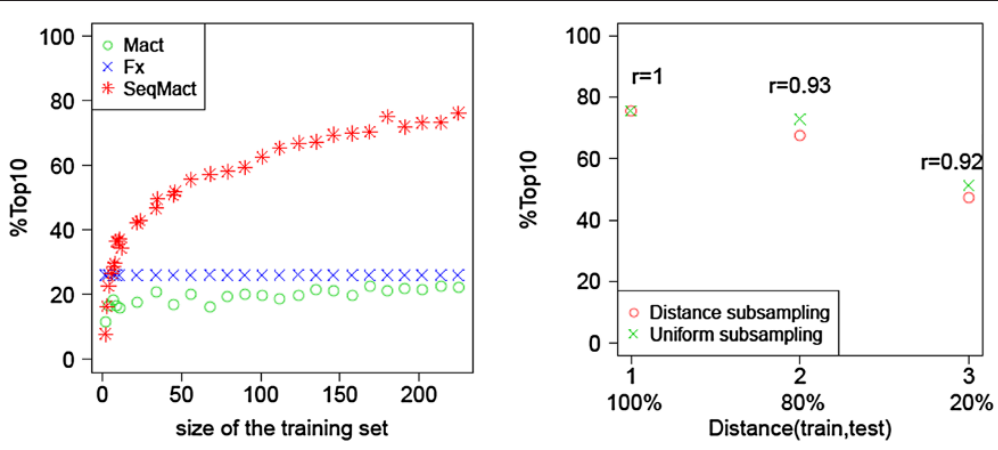

Figure 3 Performance of ML model as a function of training set composition. (Left) Performance of ML model as a function of the training set size (i.e. number of combinatorial libraries). Experimental setting are similar to those presented in Figure 2, where each point corresponds to the cross-validation performance when we use only a portion of the training data. (Right) Success rate as a function of the minimal distance between test and training targets $(1,2,3)$ - distance in number of bases, $(100 \%, 80 \%, 20 \%)$ - proportion of the training set which is kept after removal of targets which are too similar to targets in the test set. Distance subsampling - distance based selection of targets, Uniform subsampling random selection of equivalent size training set; $r$ gives the drop (ratio) in performance score due to the distance based selection of training targets. 
were any combinations of protein mutations that were harmful (or on the contrary favorable) to protein activity on any target (perhaps due to some folding or expression problems).

- SM-M2T: all features from the SM-5_11 model plus interactions between features encoding protein sequences and features encoding target sequences; this reflected the importance of dependencies between protein and target sequences, i.e. whether there were any preferences between target and protein sequences in addition to the already known cleavage activities of the p5N3 and p11N4 modules on the corresponding targets;

- SeqMact: union of all features used in previous models; the relative performance of SeqMact and SM-5_11 reflected the impact of 2nd order interactions between features encoding protein and target sequences, while the relative performance of SeqMact, SM-M2T and SM-M2M could tell us if there was any particular group of 2nd order interactions (M2M or M2T) contributing most to the performance boost.

In addition to the M2M versus M2T split, we also split 2nd order interaction features into features encoding dependencies within the same 'module' (interactions between features encoding the p5N3 part of the protein-DNA interface plus interactions between features encoding the p11N4 part) - SM-Intra, and 2nd order interaction features encoding dependencies between the p11N4 and p5N3 parts (i.e. cross-talk between combinatorial modules) - SM-Cross.

The cross-validation performance of the alternative SeqMact versions is presented in Figure 4 (Left; Top10\%, AUC and Top10 are given in Additional file 1: Figure S10). Overall, the use of information on protein sequences tripled the average number of active proteins in the top 10 predicted and the number of targets with at least one positive protein in the top 10 (SM-5_11 versus Mact). SM-5_11 basically learned which protein mutations were bad and which were good independently of the target sequence, i.e. non-specific activity patterns. The performance of SM-5_11 could be further enhanced by adding features describing simultaneous protein mutations (SM-M2M versus SM-5_11). SMM2M learned only non-specific activity patterns, but these patterns were more sophisticated: now the model also learned whether there were any particular combinations of protein mutations that were good or bad for overall performance. When we added target-specific interactions (SM-M2T), we obtained an even higher performance boost. Finally, when we combined all features (SeqMact), the result was a model that outperformed both the SM-M2T and SM-M2M models, meaning that both types of interaction were important in the final model.

The relative performance of the SM-Cross and SMIntra models suggests that the major performance boost obtained after adding interaction features came from the features describing dependencies within the same modules.

Examples of features with the strongest positive and negative impact in the model are given in Additional file 1: Table S1. The negative impact of the 44F mutation could be probably explained by the enhanced rigidity of the second beta-strand due to the contemporaneous presence of the wild type 43F; on the contrary the mutation $32 \mathrm{~K}$ could have triggered additional non-specific DNA interactions. Electrostatic repulsion between $44 \mathrm{R}$ and $77 \mathrm{R}$ may have been the cause of the negative effect of the simultaneous presence of these two mutations in the protein (Additional file 1: Figure S12 illustrates the spatial proximity of $44 \mathrm{R}$ and $77 \mathrm{R}$ leading to an important interaction between them).

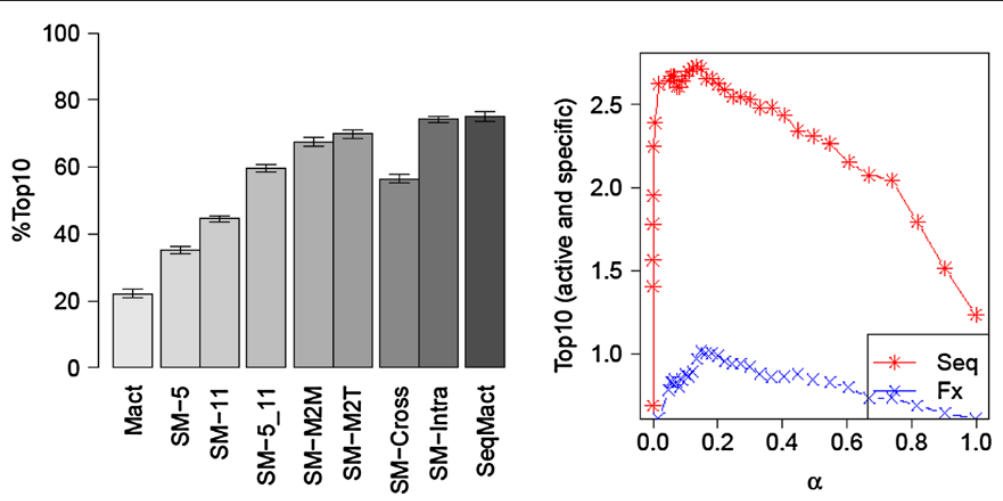

Figure 4 Cross-validation performance of $\mathbf{M L}$ model as a function of interaction features. (Left) \%Top10 - percentage of targets with at least one positive molecule in Top10 ranked. Description of various groups of features (SM-5, SM-11, SM-5_11, SM-M2M, SM-M2T, SM-Cross, SM-Intra and SeqMact) are given in the text. Error bars are estimated from 30 independent cross-validation experiments. (Right) Prediction of active mutants at least as specific as the wild type I-Crel. Top10 - avg. number of active proteins at least as specific as I-Crel in top10 ranked molecules, a - trade-off parameter between predicted specificity and activity of candidate proteins. Seq - machine learning model trained on protein/target sequences, Fx - FoldX score. 
Most of the features describing protein-DNA interactions corresponded to contacting positions, providing additional insights on which residues should or should not be used to target particular nucleotides.

\section{Activity versus specificity trade-off}

Cleavage activity on the target of interest is not the only parameter we would like to control in $\mathrm{MN}$, another important characteristic is its specificity. Since the prediction step was fast using the ML model compared to physical models, we could predict the activity of all candidate mutants on all possible targets in a matter of seconds and pick the mutant with the most specific profile or the mutant with the best trade-off between its predicted activity and specificity.

To assay experimentally a protein on all possible targets would require a tremendous amount of resources, so to validate our approach we used a set of 2,576 proteins mutated at positions contacting the $5 \mathrm{~N} 3$ region and screened them on all 64 possible $5 \mathrm{~N} 3$ targets (each mutant being active on at least one target). Screening all mutants on all targets enabled us to compute the specificity of all mutants in the same context (that of $5 \mathrm{~N} 3$ targets). Similarly to [20], we computed the specificity of mutant pi on target t $j$ as the ratio of the activity of pi on tj over the total activity of pi on all targets.

Rather than simply predicting the most active mutant, we were able to alter our criteria to favor the prediction of active mutants that were at least as specific as the wild type I-CreI on the same set of $645 \mathrm{~N} 3$ targets (other activity/specificity trade-offs are possible as well). To predict mutants that were active and specific at the same time, we first predicted the activity of the candidate mutants on all $645 \mathrm{~N} 3$ targets; we then computed the predicted specificity as the ratio of mutant activity on a given target of interest over the total activity on all 64 targets. Finally, we ranked all candidate mutants according to the following score combining predicted activity and predicted specificity:

$$
R_{\alpha}=\alpha A+(1-\alpha) S
$$

where $\mathrm{A}$ and $\mathrm{S}$ are predicted activity and specificity, respectively.

Figure 4 (Right) shows how the number of active mutants at least as specific as I-CreI in the top 10 varied as a function of $\alpha$. When we ranked the candidate mutants according to their predicted activity, we obtained only a few specific mutants $(\alpha=0)$, but when we output the most specific mutants we obtained very few active proteins. The results presented in Figure 4 were natural in the sense that if we wanted to predict not only active but also specific mutants we had to use a combined score representing a trade-off between the predicted activity and specificity of candidate mutants.

\section{De novo experiments: designing new custom MNs}

In the second series of experiments, we tested the ability of our ML model to predict MNs on completely new DNA targets. The experiments were done on two groups of targets, a first group sampled from the extended target space (ETS) (38 targets), and a second group (39 targets) sampled from the restricted target space (RTS) (a subset of ETS) (see Additional file 1: Table S2 for the exact list of targets tested). The ETS is defined by a set of constraints on the sequences of $2 \mathrm{~N} 4$ and $7 \mathrm{~N} 2$ regions and the existence of active p5N3 and p11N4 building modules, it corresponds roughly to one target every 250 base pairs. These constraints were necessary to ensure a good probability that the combinatorial approach would find an active MN [15,37]. The RTS was defined as a subset of the ETS with additional constraints on the set of $2 \mathrm{~N} 4$ sequences (only the most favorable $2 \mathrm{~N} 4$ were allowed [38]), and 11N4-5N3 combinations (with the top 20\% highest ML prediction scores), it corresponds to an average frequency of one target every $5.5 \mathrm{kbp}$.

For each target we predicted and tested 6 proteins (on average). Since the impact of $2 \mathrm{~N} 4$ regions was independent of other regions and was not taken into account in the ML model, predicted mutants were also tested on variants of sampled targets with their $2 \mathrm{~N} 4$ regions substituted by a GTAC sequence (the 2N4 sequence of the palindromic target derived from the left part of I-CreI wild type target and one of the most favorable $2 \mathrm{~N} 4$ sequences [39]). Tests on GTAC target variants enabled us to see the success rate of the ML model regarding the quality of prediction of the optimal MN binding interface independent of any influence of the $2 \mathrm{~N} 4$ region.

In the first series of experiments, $156 \mathrm{MNs}$ were predicted for 26 DNA targets sampled from the ETS. Figure 5 (Left) SeqMact presents the success rate (i.e. proportion of targets with at least one active MN) of ML predictions on the first group of targets (ETS). ORIG and GTAC denote the success rate over the original sampled targets and their GTAC variants, respectively. We also report the proportion of ORIG targets with at least one strongly active MN: ORIGstrong (cleavage activity score above 0.8 ). Overall, $62 \%(16 / 26)$ of GTAC targets and 23\% (6/26) of ORIG targets were cut. Among the six ORIG targets cut, three had strongly active MNs.

It is known [40] that additional mutations such as I132V can help boost the activity of MNs. In the second series of experiments $72 \mathrm{MNs}$ were predicted for another 18 targets sampled from the ETS, and this time all predicted MNs were synthesized with the additional mutation I132V. Figure 5 (Left) SeqMact + shows the success rate of ML predictions with additional $1132 \mathrm{~V}$ mutations. Although 

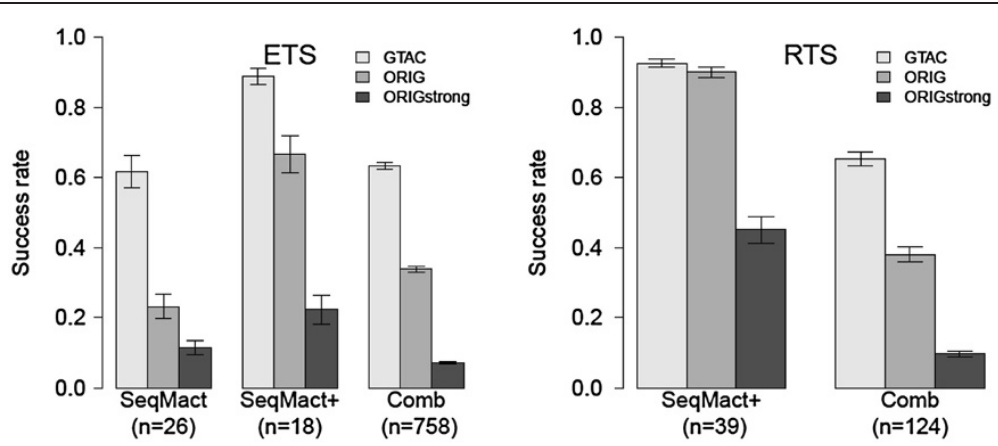

Figure 5 Success rate of meganuclease design methods. (Left) Experimental results on targets sampled from ETS (extended target space). (Right) Experimental results on targets sampled from RTS (restricted target space). SeqMact - machine learning predictions, SegMact + machine learning predictions with additional I132V mutation, Comb - combinatorial libraries. GTAC — proportion of GTAC target variants with at least one positive mutant, ORIG — proportion of original (sampled) targets with at least one positive mutant, ORIGstrong — proportion of original (sampled) targets with at least one highly active mutant (normalized cleavage activity score above 0.8).

small sample sizes did not enable us to say that there was a statistically significant difference between success rates with and without the additional I $132 \mathrm{~V}$ mutation, the proportion of successfully cut GTAC targets went up to $83 \%$, targets with original $2 \mathrm{~N} 4$ up to $58 \%$, and strongly cut original targets up to $16 \%$.

As a comparative reference, we report the success rate of the combinatorial process (Comb, high-throughput screening of about 1,160 proteins per target) on a much larger body of 758 targets also sampled from the ETS (see Figure 5 (Left) Comb). Overall, using ML predictions provided us with a success rate that was competitive in relation to that of high-throughput screening, with 200 times fewer molecules tested.

In the last series of experiments, we hypothesized that we could still improve the success rate of ML predictions by choosing targets that would be expected to be the easiest to cut [39]. To this end, 39 targets were thus randomly sampled from the more stringent RTS and assayed both with the original 2N4 and GTAC (Figure 5 (Right)). Indeed, the success rate on GTAC targets and targets with the original $2 \mathrm{~N} 4$ went up to $92 \%$ and $90 \%$ respectively ( $42 \%$ strongly cut for original $2 \mathrm{~N} 4$ ). The success rate of Comb on the RTS was the same as on the ETS.

\section{Discussion}

Apart from a significant boost in performance, relatively low performance of Mact with respect to SeqMact models provided additional evidence to the hypothesis of non-negligible interdependency between p11N4 and p5N3 protein domains [15]. Furthermore the performance of alternative ML models trained on different subsets of features (SM-Cross and SM-Intra) suggested that the principal gain in performance was coming from a better selection of starting p11N4 and p5N3 modules that keep their activity profile when combined with each other, therefore providing a basis for the desirable factorization of the binding interface.

There are several explanations for the difference in performance between the ML approach and physical models. First, physical models rely on the computation of binding energy as the only important factor to predict active MNs. However in reality there are other factors such as protein expression and cleavage activity that can greatly influence the final activity of MNs. With the ML approach we directly model the final outcome, which may be much harder to handle with a physical model. Second, existing physical models use many approximations and simplifications that significantly reduce computation time, but which may have a negative impact on precision. The majority of the computational studies predicting DNA binding affinity for MNs have used a very conservative approach, only one base has been allowed to change. In [18] a triple base change was reported. In [20] many possible reasons for the difficulty of generating new in silico MNs are clearly discussed.

Whereas the main in silico experiments with physical models were carried out using only one structure, we also studied the possibility of using more than one structure to build our models. On $20 \%$ of the original combinatorial dataset we tested the performance of the method using three different structures $(1 \mathrm{~g} 9 \mathrm{~b}, 2 \mathrm{vbj}$ and 2xe0). No detectable improvements could be reported (data not shown), suggesting that a much wider structural coverage of potential MN/DNA target pairs is necessary to improve the success rate of physical models in large scale experiments.

Concerning the application of ML approaches to the design of new DNA-binding proteins, active learning methods [41] (instead of random sampling) as a more efficient way to collect the training data is an interesting direction for future research. Such a method would be especially useful when starting data collection for a new 
binding scaffold since it would help to choose the most informative examples for sampling and to reach faster the optimal success rate.

\section{Conclusions}

In this study we address the application of a machine learning approach to the design of meganucleases cutting specific DNA targets. Our results are very promising in that the ML model significantly outperformed state-of-the-art in silico models such as FoldX and Rosetta. In addition, our method had success rates competing with those of combinatorial high-throughput screening, while reducing the number of molecules screened by several orders of magnitude. In experimental validation, the ML model successfully predicted active MNs for 53 new DNA targets. The boost in performance brought by the ML model comes with a price, as one needs a training set to learn the model, meaning it cannot be used completely $a b$ initio. However, if a training set already exists, or if one is prepared to invest in building a new one, this could be considered a very interesting alternative to existing methods. In the case of MNs, experimental results on 20 DNA targets were already enough to train a machine learning model that outperformed existing in silico models.

\section{Methods}

\section{Combinatorial process}

A detailed description of the combinatorial process can be found in [17]. Here, we give a brief description of the procedure according to which the dataset of combinatorial libraries was generated. The goal of the combinatorial process was to find MNs that cut a given $22 \mathrm{bp}$ DNA target $\mathrm{T}$. We consider the case of palindromic target $\mathrm{T}$ (the case of a non-palindromic target can easily be reduced to the palindromic case by designing a separate meganuclease for the left and right parts of T; see [17]). The preliminary step in the combinatorial process involved creating module libraries of mutants cutting all possible 5N3 targets (64 targets) and all possible 11N4 targets (256 targets). Module libraries were created by degenerating a pool of I-CreI mutants at positions Q44, R68, R70, D75, I77 (hereinafter denoted by $\mathrm{p} 5 \mathrm{~N} 3$ ) contacting $5 \mathrm{~N} 3$ regions and positions N30, S32, Y33, Q38, S40 (hereinafter denoted by p11N4) contacting $11 \mathrm{~N} 4$ regions with further screening on all possible 5N3 (respectively 11N4) targets. This preliminary step needed to be done only once for all future targets. Since the sets of I-CreI interface residues contacting $5 \mathrm{~N} 3$ and 11N4 regions were disjoint, a $\mathrm{MN}$ combining mutations of $\mathrm{MNs}$ cutting the $5 \mathrm{~N} 3$ and $11 \mathrm{~N} 4$ targets had a good chance of cutting the combined 11N4-5N3 target. In practice, testing a single combined $\mathrm{MN}$ rarely leads to success, and in order to have a good probability of finding an active $\mathrm{MN}$ up to 40 by 40 combinations (i.e. 1,600 combinations of 40 p5N3
MNs and 40 p11N4 MNs) have to be tested in highthroughput screening.

\section{Dataset of combinatorial libraries}

When applying the combinatorial process to find active MNs for a given palindromic target of interest $\mathrm{T}$, we started from the selection of $\mathrm{MN}$ modules targeting the $5 \mathrm{~N} 3$ and $11 \mathrm{~N} 4$ part of $\mathrm{T}$, which were later used in the PCR assembly process (see "Combinatorial process") to generate a number of final MNs p1,..,pK where pi is a combination of p5N3 mutations of a random $\mathrm{p} 5 \mathrm{~N} 3$ module and p11N4 mutations of a random p11N4 module. After screening candidate mutants $\mathrm{p} 1, \ldots, \mathrm{pK}$ on the target of interest $\mathrm{T}$, only positive mutants (if any) were sequenced. Although due to the random nature of the PCR assembly process we could not guarantee that all possible combinations of starting modules were present among the $\mathrm{K}$ candidate mutants, we could make the number of untested mutants quite low by oversampling (in our lab we sampled triple the theoretical diversity which should cover about $95 \%$ of all possible combinations). It was therefore natural to assume that 11N4$5 \mathrm{~N} 3$ combinations, which are not found among positive sequences, were negative.

The dataset used in our ML computations consisted of 251 palindromic targets, all with GTAC (2N4 sequences of the palindromic target derived from the left part of I-CreI wild type target [39]) at their 2N4 region, screened according to the combinatorial process. This set was used to train/cross-validate the machine learning model and to compare the "cross-validation performance" of various in silico models. The average size of the combinatorial pools was 1,160 mutants. 26 targets had associated combinatorial pools with less than 500 mutants and 10 targets had pools of 2,000 or more mutants. The average number of positive mutants per target was 15 , and 27 targets had only one positive mutant (all targets had at least one positive mutant). 19 targets had more than 40 associated positive mutants. Since only positive mutants were sequenced at the end of the combinatorial process, we could only be sure about the sequences of active proteins. The rest of the combinatorial pools are likely to have been negative and are considered, but not guaranteed, as such.

\section{N3 cross-validation set}

The set of p5N3 mutants used to evaluate the "activity versus specificity trade-off" (see Section 3.3) consisted of 2,576 mutants tested on all 64 possible 5N3 DNA targets. 11,013 out of $164,864(=64 * 2,576)$ protein-target pairs (6.6\%) were positive, giving on average 172 positive mutants per target and 4 positive targets per mutant.

\section{Cross-validation performance scores}

As explained above, the dataset consisted of a set of DNA targets $t_{i}$ and associated candidate proteins $\mathrm{p}_{\mathrm{ij}}$. We 
used in silico methods to compute an activity score $\mathrm{A}\left(\mathrm{t}_{\mathrm{i}}\right.$, $\mathrm{p}_{\mathrm{ij}}$ ) which was then used to rank candidate proteins $\mathrm{p}_{\mathrm{i} 1} \ldots$ $\mathrm{p}_{\mathrm{iK}}$ for a given target $t_{\mathrm{i}}$. A perfect score would put all active proteins at the top of the list, but in practice we usually had a mix of positive and negative proteins, with the proportion of active proteins depending on the quality of the scoring function. To assess the quality of a ranking generated by a particular score, we used the following measures:

- \%TopN - indicated whether there is at least one active molecule among the top $\mathrm{N}$ predicted; this score makes sense only when averaged over a set of targets; in this case it represents the percentage of targets where an in silico method was able to predict at least one positive in the top $\mathrm{N}$ (i.e. a successful experiment);

- TopN - in practice an in silico model is usually used to select a limited number of candidate molecules that will later be tested in real experiments. In this context, we used the number of active molecules among the top $\mathrm{N}$ predicted as a performance measure. In most of the figures, we used $\mathrm{N}=10$, but relative behavior of in silico methods was quite stable over various values of $\mathrm{N}$ (see "Cross-validation experiments" Additional file 1: Figure S1);

- AUC - area under the ROC (receiver operating characteristic) curve, a popular measure of global ranking quality [42]; one of the interpretations of this score is the probability that a random positive element (meganuclease in our case) will be ranked higher than a random negative element from the list of candidate proteins;

\section{ML model}

The final machine learning model was an ensemble model combining GBM (gradient boosting machines with decision trees as basic learning models) [43] and LASSO (least absolute shrinkage and selection operator) models [44]. We used the gbm package [45] to train the GBM model and the glmnet package [44] to train the LASSO model. Classical SVM (hinge loss, L2 regularization) showed inferior performance, but rankSVM model (optimization of the AUC score) was competitive with LASSO and GBM. A variant of the GBM model based on the optimization of the AUC score lead to a slight improvement in terms of AUC, but had a rather negative impact on Top10 and \%Top10. The final ensemble combination was restricted only to LASSO and GBM, addition of other models had no significant impact on the performance of the ensemble model. Parameters of ML models were systematically estimated from inner cross-validation loops on training folds (see Additional file 1: Figure S2) and then used to test the final model on the test fold. Additional file 1: Figure S3 shows the performance of these models separately and when combined together in an ensemble model. A detailed description of the algorithm used to build the ensemble model is given in Additional file 1: Figure S11.

GBM was trained on categorical features representing mutations at 11 key positions in the protein sequence, the DNA target sequence and cleavage activities of starting p5N3 and p11N4 building modules (a detailed description of the dataset is given in Additional file 1: Figure S4 "Categorical dataset"). To train the LASSO model, all categorical features were encoded using binary features (with each binary feature encoding a particular mutation of the protein sequence or nucleotides in the DNA sequence). In addition, products of all pairs of individual features with more than 200 non-zero components were added to the model to encode second-order interactions between features (detailed description of the datasets is given in Additional file 1: Figure S4 "Bin1" and "Bin2" dataset).

\section{Endnote}

${ }^{\mathrm{a}} \mathrm{TALEN}^{\mathrm{m}}$ is a trademark owned by Cellectis bioresearch.

\section{Additional file}

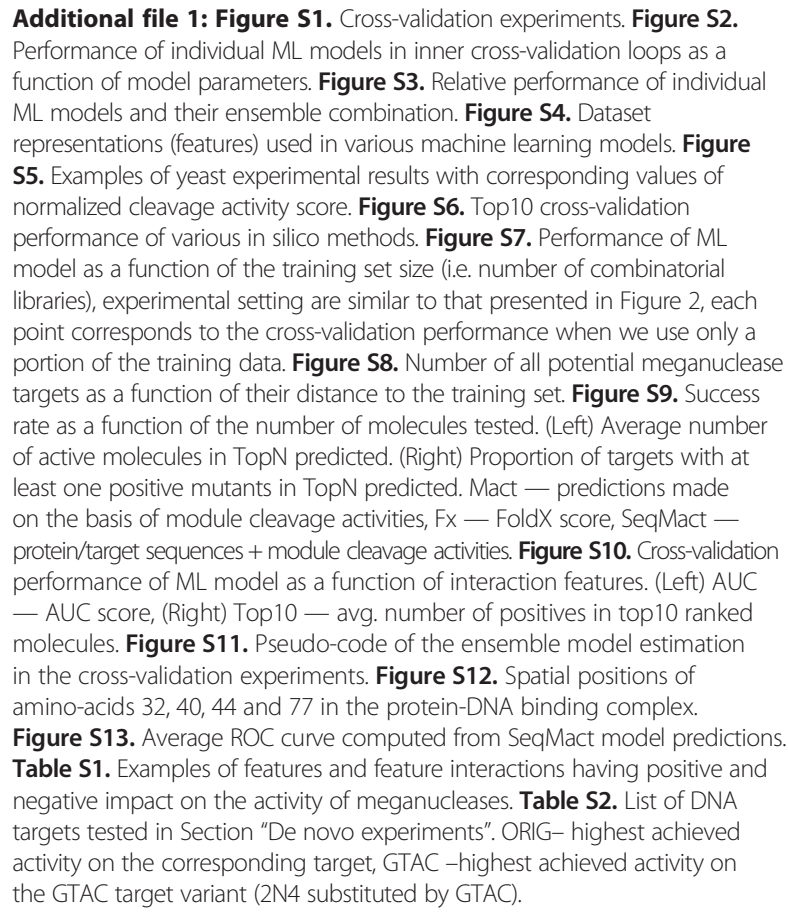




\section{Authors' contributions}

MZ performed the data processing, built the machine learning model, participated in the design of the study and drafted the manuscript. CB carried out computational biology experiments, participated in the design of the study and drafted the manuscript. AD coordinated the data processing, participated in the design of the study and drafted the manuscripts. PD and GS conceived of the study, participated in its design and coordination and helped to draft the manuscript. All authors read and approved the final manuscript.

\section{Acknowledgements}

We would like to thank F.Paques, J-C. Epinat and F.Cedrone for their many insightful discussions. We acknowledge the significant contribution of the Cellectis Nuclease Production Platform for providing meganuclease reagents and experimental support.

Received: 21 January 2014 Accepted: 3 June 2014

Published: 17 June 2014

\section{References}

1. Umezawa T, Fujita M, Fujita Y, Yamaguchi-Shinozaki K, Shinozaki K Engineering drought tolerance in plants: discovering and tailoring genes to unlock the future. Curr Opin Biotechnol 2006, 17(2):113-122.

2. Lee SK, Chou H, Ham TS, Lee TS, Keasling JD: Metabolic engineering of microorganisms for biofuels production: from bugs to synthetic biology to fuels. Curr Opin Biotechnol 2008, 19(6):556-563.

3. Silva G, Poirot L, Galetto R, Smith J, Montoya G, Duchateau P, Paques F: Meganucleases and other tools for targeted genome engineering: perspectives and challenges for gene therapy. Curr Gene Ther 2011, 11(1):11-27.

4. Boch J, Scholze H, Schornack S, Landgraf A, Hahn S, Kay S, Lahaye T, Nickstadt A, Bonas U: Breaking the code of DNA binding specificity of TAL-type III effectors. Science 2009, 326(5959):1509-1512.

5. Jiang W, Bikard D, Cox D, Zhang F, Marraffini LA: RNA-guided editing of bacterial genomes using CRISPR-Cas systems. Nat Biotechnol 2013, 31(3):233-239.

6. Mali P, Yang L, Esvelt KM, Aach J, Guell M, DiCarlo JE, Norville JE, Church GM: RNA-guided human genome engineering via Cas9. Science 2013, 339(6121):823-826.

7. Urnov FD, Rebar EJ, Holmes MC, Zhang HS, Gregory PD: Genome editing with engineered zinc finger nucleases. Nat Rev Genet 2010, 11(9):636-646.

8. Townsend JA, Wright DA, Winfrey RJ, Fu F, Maeder ML, Joung JK, Voytas DF: High-frequency modification of plant genes using engineered zinc-finger nucleases. Nature 2009, 459(7245):442-445.

9. Arnould S, Delenda C, Grizot S, Desseaux C, Paques F, Silva GH, Smith J: The I-Crel meganuclease and its engineered derivatives: applications from cell modification to gene therapy. Protein Eng Des Sel 2011, 24(1-2):27-31.

10. Daboussi F, Zaslavskiy M, Poirot L, Loperfido M, Gouble A, Guyot V, Leduc S, Galetto R, Grizot S, Oficjalska D, Perez C, Delacote F, Dupuy A, Chion-Sotinel I, Le Clerre D, Lebuhotel C, Danos O, Lemaire F, Oussedik O, Cedrone F, Epinat JC, Smith J, Dickson G, Popplewell L, Koo T, VandenDriessche T, Chuah M, Duclert A, Duchateau P, Paques F: Chromosomal context and epigenetic mechanisms control the efficacy of genome editing by rare-cutting designer endonucleases. Nucleic Acids Res 2012, 40(13):6367-6379.

11. Pingoud A, Silva GH: Precision genome surgery. Nat Biotechnol 2007, 25(7):743-744

12. Baxter S, Lambert AR, Kuhar R, Jarjour J, Kulshina N, Parmeggiani F, Danaher P, Gano J, Baker D, Stoddard BL, Scharenberg AM: Engineering domain fusion chimeras from I-Onul family LAGLIDADG homing endonucleases. Nucleic Acids Res 2012, 40(16):7985-8000.

13. Grizot S, Epinat JC, Thomas S, Duclert A, Rolland S, Paques F, Duchateau P: Generation of redesigned homing endonucleases comprising DNAbinding domains derived from two different scaffolds. Nucleic Acids Res 2009, 38(6):2006-2018.

14. Epinat JC, Arnould S, Chames P, Rochaix P, Desfontaines D, Puzin C, Patin A, Zanghellini A, Paques F, Lacroix E: A novel engineered meganuclease induces homologous recombination in yeast and mammalian cells. Nucleic Acids Res 2003, 31(11):2952-2962.

15. Grizot S, Duclert A, Thomas S, Duchateau P, Paques F: Context dependence between subdomains in the DNA binding interface of the I-Crel homing endonuclease. Nucleic Acids Res 2011, 39(14):6124-6136.
16. Arnould S, Chames P, Perez C, Lacroix E, Duclert A, Epinat JC, Stricher F, Petit AS, Patin A, Guillier S, Rolland S, Prieto J, Blanco FJ, Bravo J, Montoya G, Serrano L, Duchateau P, Paques F: Engineering of large numbers of highly specific homing endonucleases that induce recombination on novel DNA targets. J Mol Biol 2006, 355(3):443-458.

17. Smith J, Grizot S, Arnould S, Duclert A, Epinat JC, Chames P, Prieto J, Redondo P, Blanco FJ, Bravo J, Montoya G, Paques F: A combinatorial approach to create artificial homing endonucleases cleaving chosen sequences. Nucleic Acids Res 2006, 34(22):e149.

18. Ashworth J, Taylor GK, Havranek JJ, Quadri SA, Stoddard BL, Baker D: Computational reprogramming of homing endonuclease specificity at multiple adjacent base pairs. Nucleic Acids Res 2010, 38(16):5601-5608.

19. Schymkowitz J, Borg J, Stricher F, Nys R, Rousseau F, Serrano L: The FoldX web server: an online force field. Nucleic Acids Res 2005, 33:W382-W388.

20. Ulge UY, Baker DA, Monnat RJ Jr: Comprehensive computational design of $\mathrm{mCrel}$ homing endonuclease cleavage specificity for genome engineering. Nucleic Acids Res 2011, 39(10):4330-4339.

21. Ashworth J, Havranek JJ, Duarte CM, Sussman D, Monnat RJ Jr, Stoddard BL, Baker D: Computational redesign of endonuclease DNA binding and cleavage specificity. Nature 2006, 441(7093):656-659.

22. Jarjour J, West-Foyle H, Certo MT, Hubert CG, Doyle L, Getz MM, Stoddard BL, Scharenberg AM: High-resolution profiling of homing endonuclease binding and catalytic specificity using yeast surface display. Nucleic Acids Res 2009, 37(20):6871-6880

23. Doyon JB, Pattanayak V, Meyer CB, Liu DR: Directed evolution and substrate specificity profile of homing endonuclease I-Scel. J Am Chem Soc 2006 128(7):2477-2484.

24. Seligman LM, Chisholm KM, Chevalier BS, Chadsey MS, Edwards ST, Savage $J H$, Veillet AL: Mutations altering the cleavage specificity of a homing endonuclease. Nucleic Acids Res 2002, 30(17):3870-3879.

25. Sussman D, Chadsey M, Fauce S, Engel A, Bruett A, Monnat R Jr, Stoddard $\mathrm{BL}$, Seligman LM: Isolation and characterization of new homing endonuclease specificities at individual target site positions. J Mol Biol 2004, 342(1):31-41.

26. Stoddard BL: Homing endonuclease structure and function. Q Rev Biophys 2005, 38(1):49-95.

27. Berman HM: The Protein Data Bank: a historical perspective. Acta Crystallogr A 2008, 64(Pt 1):88-95.

28. Murzin AG, Brenner SE, Hubbard T, Chothia C: SCOP: a structural classification of proteins database for the investigation of sequences and structures. J Mol Biol 1995, 247(4):536-540.

29. Chevalier BS, Monnat RJ Jr, Stoddard BL: The homing endonuclease I-Crel uses three metals, one of which is shared between the two active sites. Nat Struct Biol 2001, 8(4):312-316.

30. Jansen $\mathrm{R}, \mathrm{Yu} \mathrm{H}$, Greenbaum D, Kluger Y, Krogan NJ, Chung S, Emili A, Snyder M, Greenblatt JF, Gerstein M: A Bayesian networks approach for predicting protein-protein interactions from genomic data. Science 2003, 302(5644):449-453.

31. Ben-Hur A, Noble WS: Kernel methods for predicting protein-protein interactions. Bioinformatics 2005, 21(1):i38-i46.

32. Cai $C Z$, Wang $W L$, Sun $L Z$, Chen $Y Z$ : Protein function classification via support vector machine approach. Math Biosci 2003, 185(2):111-122.

33. Borgwardt KM, Ong CS, Schonauer S, Vishwanathan SV, Smola AJ, Kriegel HP: Protein function prediction via graph kernels. Bioinformatics 2005, 21(1):i47-i56.

34. Qian J, Lin J, Luscombe NM, Yu H, Gerstein M: Prediction of regulatory networks: genome-wide identification of transcription factor targets from gene expression data. Bioinformatics 2003, 19(15):1917-1926.

35. Mordelet F, Vert JP: SIRENE: supervised inference of regulatory networks. Bioinformatics 2008, 24(16):i76-i82.

36. Margolin AA, Nemenman I, Basso K, Wiggins C, Stolovitzky G, Dalla Favera R, Califano A: ARACNE: an algorithm for the reconstruction of gene regulatory networks in a mammalian cellular context. BMC Bioinformatics 2006, 7(1):S7.

37. Voziyanov $Y$, Konieczka JH, Stewart AF, Jayaram M: Stepwise manipulation of DNA specificity in Flp recombinase: progressively adapting Flp to individual and combinatorial mutations in its target site. J Mol Biol 2003 326(1):65-76.

38. Molina R, Redondo P, Stella S, Marenchino M, D'Abramo M, Gervasio FL, Epinat JC, Valton J, Grizot S, Duchateau P, Prieto J, Montoya G: Non-specific protein-DNA interactions control I-Crel target binding and cleavage. Nucleic Acids Res 2012, 40(14):6936-6945. 
39. Chevalier B, Turmel M, Lemieux C, Monnat RJ Jr, Stoddard BL: Flexible DNA target site recognition by divergent homing endonuclease isoschizomers I-Crel and I-Msol. J Mol Biol 2003, 329(2):253-269.

40. Gouble A, Grizot S, Perez-Michaut C: Method for enhancing the cleavage activity of i-crei derived meganucleases. US Patent App. 12/663,445; 2008.

41. Warmuth MK, Liao J, Ratsch G, Mathieson M, Putta S, Lemmen C: Active learning with support vector machines in the drug discovery process. J Chem Inf Comput Sci 2003, 43(2):667-673.

42. Fawcett T: An introduction to ROC analysis. Pattern Recogn Lett 2006, 27(8):861-874.

43. Friedman JH: Stochastic gradient boosting. Comput Stat Data Anal 2002, 38(4):367-378.

44. Friedman J, Hastie T, Tibshirani R: Regularization Paths for Generalized Linear Models via Coordinate Descent. J Stat Softw 2010, 33(1):1-22.

45. Ridgeway G: gbm: Generalized Boosted Regression Models. R package manual 2010, http://cran.r-project.org/web/packages/gbm/.

doi:10.1186/1471-2105-15-191

Cite this article as: Zaslavskiy et al:: Efficient design of meganucleases using a machine learning approach. BMC Bioinformatics 2014 15:191.

\section{Submit your next manuscript to BioMed Central and take full advantage of:}

- Convenient online submission

- Thorough peer review

- No space constraints or color figure charges

- Immediate publication on acceptance

- Inclusion in PubMed, CAS, Scopus and Google Scholar

- Research which is freely available for redistribution 